\title{
Determination of Mineralogical Contents of a Bangle Shard from an Indus Valley Settlement through X-ray Diffraction Analysis
}

\author{
Saheeb Ahmed Kayani \\ National University of Sciences and Technology, \\ Islamabad, Pakistan
}

\author{
Rehan ul Haq Siddiqui \\ Geoscience Advance Research Laboratories \\ Geological Survey of Pakistan, \\ Islamabad, Pakistan
}

The mineralogical make up of clay used in a bangle shard (Fig. 1) collected from Harrappa, an Indus Valley settlement now located in Pakistan has been determined using XRD testing carried out on 'Philips X'Pert PRO X-ray diffractometer' available in Geoscience Advance Research Laboratories (a facility of Geological Survey of Pakistan) in Islamabad. XRD analysis shows that the mineralogical make up includes montmorillonite $\left((\mathrm{Na}, \mathrm{Ca})_{0.33}(\mathrm{Al}, \mathrm{Mg})_{2}\left(\mathrm{Si}_{4} \mathrm{O}_{10}\right)(\mathrm{OH})_{2} \cdot \mathrm{nH}_{2} \mathrm{O}\right)$, gypsum $\left(\mathrm{CaSO}_{4} \cdot 2 \mathrm{H}_{2} \mathrm{O}\right)$, and quartz $\left(\mathrm{SiO}_{2}\right)$. The X-ray diffractogram was developed using PW3050/60 goniometer. The generator settings were $30 \mathrm{~mA} / 40 \mathrm{kV}$ with CuKa radiation of $\lambda=1.5406 \AA$. Continuous scanning was carried out from 10.01 as $2 \theta$ start position to 79.99 as $2 \theta$ end position, with a step size of 0.02 and step time of $1 \mathrm{~s}$.

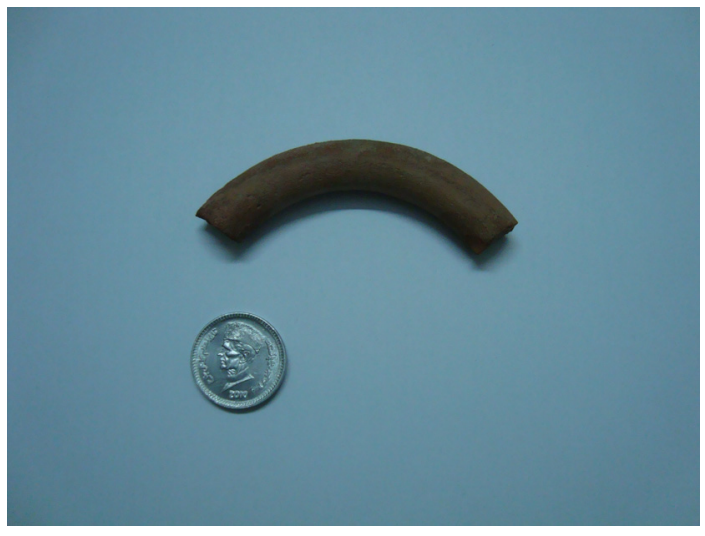

Fig. 1 Bangle shard photographed with Pakistani one rupee coin as reference. Diameter: $0.95-1 \mathrm{~cm}$, length: $5.65 \mathrm{~cm}$ or about $2 / 5^{\text {th }}$ of the complete bangle.

XRD Testing Parameters and Results

\section{1(a) Anchor Scan Parameters}

Dataset Name:

File Name:

Comment:
$\mathrm{H}-1$

C:IX'Pert Datal2011|MaylH-1.xrdml

Material analysis for universities

Configuration=Flat Sample Stage, Owner=User-1, Creation

date=2/5/2008 9:32:46 AM

Goniometer=PW3050/60 (Theta/Theta); Minimum step size

2Theta:0.001; Minimum step size Omega:0.001

Sample stage $=P W 3071 / x x$ Bracket 


\section{Diffractometer system=XPERT-PRO}

Measurement program=material analysis, Owner=User -1 , Creation date $=3 / 28 / 2008$ 10:27:40 AM

synthetic material

Measurement Date / Time:

5/6/2011 9:25:21 PM

Operator:

Geoscience

Raw Data Origin:

XRD measurement ( $\left.{ }^{*} . \mathrm{XRDML}\right)$

Scan Axis:

Gonio

Start Position [ ${ }^{\circ} 2$ Th.]:

10.0100

End Position [ ${ }^{2}$ Th.]:

79.9900

Step Size [ $\left.{ }^{\circ} 2 T h.\right]$ :

0.0200

Scan Step Time [s]:

1.0000

Scan Type:

Continuous

Offset [ ${ }^{\circ} 2 \mathrm{Th}$.]:

0.0000

Divergence Slit Type:

Fixed

Divergence Slit Size $\left[{ }^{\circ}\right]$ :

0.5000

Specimen Length [mm]:

10.00

Receiving Slit Size [mm]:

0.1000

Measurement Temperature $\left[{ }^{\circ} \mathrm{C}\right]$ :

Anode Material:

25.00

K-Alpha1 [Å]:

1.54060

K-Alpha2 [Å]:

1.54443

K-Beta $[\AA ̊]$ :

1.39225

K-A2 / K-A1 Ratio:

0.50000

Generator Settings:

$30 \mathrm{~mA}, 40 \mathrm{kV}$

Diffractometer Type:

0000000011037156

Diffractometer Number:

0

Goniometer Radius [mm]: $\quad 240.00$

Dist. Focus-Diverg. Slit [mm]: $\quad 100.00$

Incident Beam Monochromator: No

Spinning:

No

1(b) Graphics

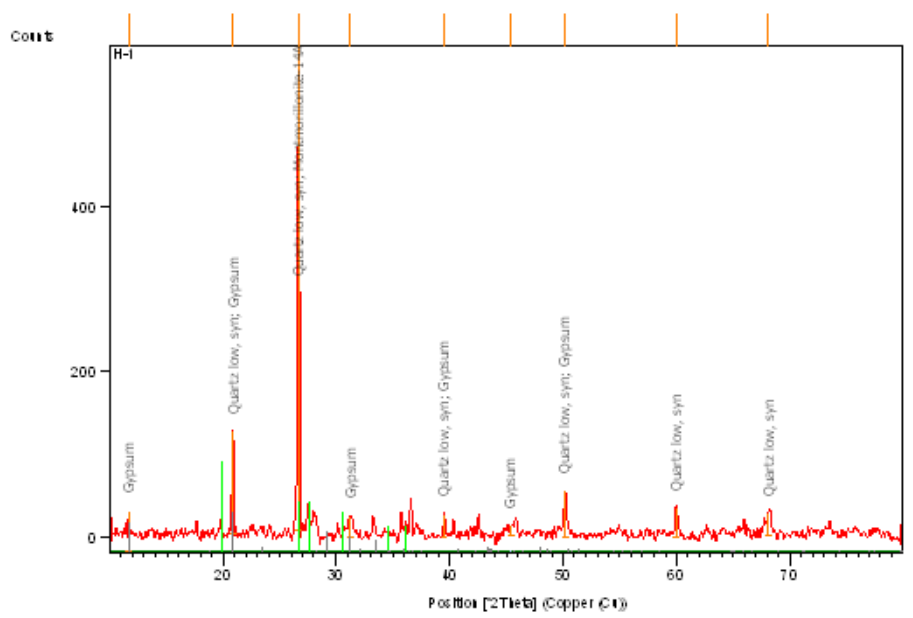




\section{2(a) Peak List}

$\begin{array}{ccccccr}\text { Pos.["2Th.] } & \text { Height[cts] } & \text { FWHM[ }{ }^{\circ} \text { 2Th.] } & \text { d-spacing[A] } & \text { Rel.Int.[\%] } & \text { Tipwidth[ }{ }^{\circ} \text { 2Th.] } & \text { Matched by } \\ 11.6307 & 48.56 & 0.0150 & 7.60871 & 8.23 & 0.0180 & 01-072-0596 \\ 20.8542 & 124.13 & 0.1771 & 4.25968 & 21.05 & 0.2125 & 03-065-0466 ; 01 . . \\ 26.6610 & 589.75 & 0.1968 & 3.34364 & 100.00 & 0.2362 & 03-065-0466 ; 00 . . \\ 31.2494 & 25.46 & 0.4723 & 2.86237 & 4.32 & 0.5668 & 01-072-0596 \\ 39.5060 & 28.61 & 0.2362 & 2.28111 & 4.85 & 0.2834 & 03-065-0466 ; 01 . . \\ 45.3856 & 11.54 & 0.9446 & 1.99833 & 1.96 & 1.1336 & 01-072-0596 \\ 50.2031 & 55.70 & 0.1968 & 1.81729 & 9.44 & 0.2362 & 03-065-0466 ; 01 . . \\ 59.9984 & 35.04 & 0.2362 & 1.54191 & 5.94 & 0.2834 & 03-065-0466 \\ 68.1453 & 27.77 & 0.5760 & 1.37494 & 4.71 & 0.6912 & 03-065-0466\end{array}$

\section{2(b) Pattern List}

\begin{tabular}{|c|c|c|c|c|c|c|}
\hline Visible & Ref. Code & Score & Compound Name & Displ..[2Th] & Scale Fac. & Chem. Formula \\
\hline & 03-065-0466 & 72 & Quartz low, syn & 0.000 & 0.962 & $\mathrm{SiO} 2$ \\
\hline * & 00-013-0259 & 5 & Montmorillonite-14A & 0.000 & 9.948 & $\mathrm{Na} 0.3(\mathrm{Al}, \mathrm{Mg}) 2$. \\
\hline * & 01-072-0596 & 11 & Gypsum & 0.000 & 0.077 & $\mathrm{Ca}(\mathrm{SO} 4)(\mathrm{H} 2 \mathrm{O}$. \\
\hline
\end{tabular}

2(c) Graphics

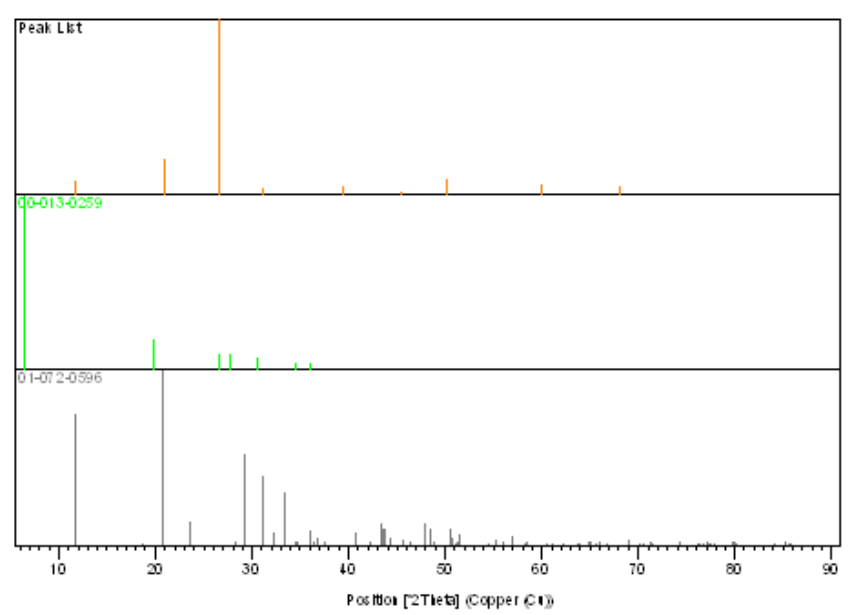

